Europhysics Letters

PREPRINT

\title{
Diffusion mechanisms of localised knots along a polymer
}

\author{
Ralf Metzler ${ }^{12}\left(^{*}\right)$, Walter Reisner ${ }^{3}$, Robert Riehn ${ }^{4}$, Robert Austin ${ }^{4}$, Jo- \\ NAS Tegenfeldt ${ }^{5}$, and Igor M. Sokolov ${ }^{6}$ \\ 1 NORDITA - Blegdamsvej 17, DK-2100 Copenhagen \\ 2 New address: Physics Dept, University of Ottawa-Ottawa, ON, K1N 6N5, Canada \\ 3 Biophysics Dept, Risø National Laboratory, Frederiksborgvej 399, DK-4000 Roskilde \\ 4 Department of Physics, Princeton University, Princeton, NJ 08544 \\ 5 Division of Solid State Physics, Lund University, Sölvegatan 14, S-223 62 Lund \\ 6 Inst. für Physik, Humboldt Universität zu Berlin - Newtonstraße 15, D-12489 Berlin
}

PACS. 87.14.Gg - DNA, RNA.

PACS. 02.50.Ey - Stochastic processes.

PACS. 82.37.-j - Single molecule kinetics.

\begin{abstract}
We consider the diffusive motion of a localized knot along a linear polymer chain. In particular, we derive the mean diffusion time of the knot before it escapes from the chain once it gets close to one of the chain ends. Self-reptation of the entire chain between either end and the knot position, during which the knot is provided with free volume, leads to an $L^{3}$ scaling of diffusion time; for sufficiently long chains, subdiffusion will enhance this time even more. Conversely, we propose local "breathing", i.e., local conformational rearrangement inside the knot region (KR) and its immediate neighbourhood, as additional mechanism. The contribution of KR-breathing to the diffusion time scales only quadratically, $\sim L^{2}$, speeding up the knot escape considerably and guaranteeing finite knot mobility even for very long chains.
\end{abstract}

Introduction. - Double-stranded DNA under standard salt conditions has a persistence length $\ell_{p}$ of circa $50 \mathrm{~nm}$. Nanochannels can presently be manufactured with widths that are comparable to, or even smaller than, $\ell_{p}$. In such channels, that is, linear DNA is stretched beyond the blob regime, assuming undulations in the bending-affected Odijk regime [1]. This makes it possible to detect and separate individual DNA molecules and characterise, for instance, their length $[2,3]$; it even appears feasible in the future to use nanochannels for sequence determination [4]. While being pushed through the nanochannel by an electric field, the DNA chain occasionally collides with local channel defects, bottlenecks created during the manufacturing process. Such collisions often cause a superstructure in the chain, visible in the experiment using stained DNA as a spot of increased fluorescence and concurrent reduction of the end-to-end length of the chain (figure 11). On switching off the electric field, after a period of immobilisation, these features eventually move toward one of the chain ends and escape [5]. We argue that these intermittent features likely correspond to knots threaded into the DNA

$\left(^{*}\right)$ E-mail: metz@nordita.dk

(c) EDP Sciences 

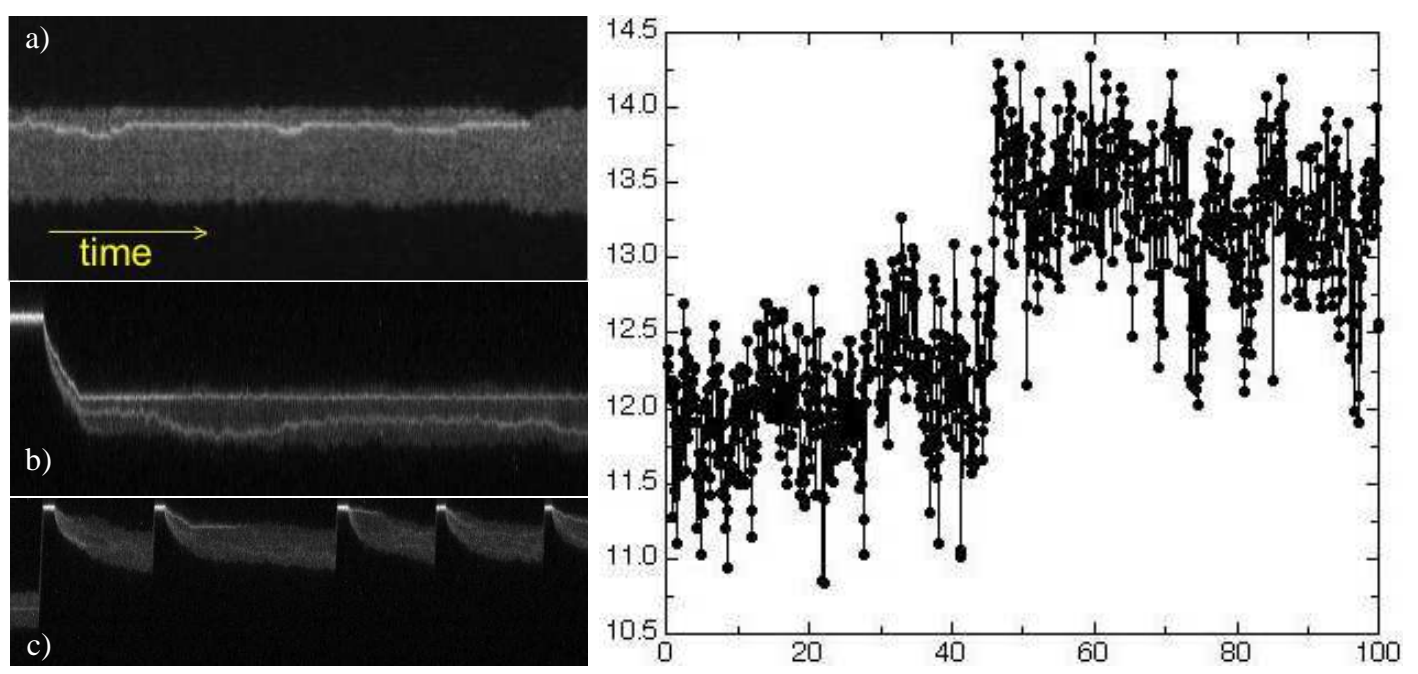

Fig. 1 - Phage $\lambda$ DNA of contour length $L \approx 16.5 \mu \mathrm{m}$ in a $60 \times 80 \mathrm{~nm}$ channel. Left: Three fluorescence time-traces of the DNA. a) The feature is almost immobile before escape; b) Two features created by a forced collision; c) repeated collisions producing new features, that escape prior to a new collision. Right: Evolution $([\mu \mathrm{m}]$ versus [sec] $)$ of the end-to-end length corresponding to the trace in a). The jump $(\Delta N \approx 2.3 \mu \mathrm{m}$, or $6.8 \mathrm{kbp})$ at around $42 \mathrm{sec}$ corresponds to the escape of the feature [5].

on collision. $\left({ }^{1}\right)$ In what follows, we propose a mechanism for the diffusive escape of the knot, obtaining a shorter than previously assumed knot diffusion time.

Knots also occur in vivo. For instance, in bacteria cells DNA knots are created physiologically [6]; they are implicated to have an active role in gene regulation, separating topologically different parts of the genome [7]. Packaging of mutant DNA into virus capsids turns out to be a highly efficient manner to produce DNA knots in vitro [8]. After dissociation of the capsid, the knot needs to be locked by cyclisation of the formerly linear DNA, before it escapes at either chain end. In both cases it is of interest to characterise better the knot motion along the DNA chain. Nanochannel techniques promise to add novel quantitative information to the understanding of knot motion in polymers.

Knot diffusion in stretched, single $\lambda$ DNA was studied experimentally by Bao et al. [9]. In their analysis they argue that the diffusion time of the knot from an initial position inside the chain to one of the ends should scale like $L^{2} / D$. Assuming a diffusion coefficient $D \simeq L^{-1}$, the diffusion time $\tau_{D}$ of the knot scales like $\tau_{D} \simeq L^{3}$. This scenario implies that the entire chain self-reptates to create the motion of the knot. We here argue that (i) this self-reptation is asymmetric in an open knot leading to increase mobility toward the chain ends, and (ii) suggest an additional mechanism, namely, breathing of the knot region $(\mathrm{KR})\left({ }^{2}\right)$. KR-breathing involves exchange of stored length locally with the vicinal chain. This mechanism is expected to dominate in long chains, significantly increasing the knot diffusivity with quadratic scaling of the diffusion time, $\tau_{D} \simeq L^{2}$. Even in long chains, KR-breathing causes Brownian diffusion.

Let us first discuss the nature of the observed feature. A priori, one might suspect that the feature is a fold $\left({ }^{3}\right)$ in the DNA of the shape of a $\mathrm{Z}$ or a $\mathrm{Q}$, or a knot. Both simple knot

$\left({ }^{1}\right)$ Repeated collision of the same chain with the defect almost always causes a new superstructure (figure 1).

$\left({ }^{2}\right)$ In the case of a localized knot, the volume that contains all non-trivial topological entanglements [10].

$\left({ }^{3}\right)$ Similar to folds created when trying to push an electric cable in a larger cable canal. 
and fold structures carry a bending energy amounting to some $\left.3 k_{B} T .{ }^{4}\right)$ This is a fairly small energy such that thermal fluctuations would be sufficient to remove the folds. Moreover, the folds are not self-confining so that they should fluctuate significantly in size; on the images, however, the fluorescence spot is strongly localized at all times. In fact, it is known that in nano-confined DNA knotting occurs almost with probability one [8]. We are therefore confident that the observed features correspond to a knotted state of the DNA chain. While the knotting studies in nano-confinement suggest that the created knots are more complex, a defect size of $\approx 2 \mu \mathrm{m}$ indeed suggests a knot with larger number of essential crossings $[9,11]$.

Immobilisation period. - Once formed while being smashed against the channel defect by the external electric field, the created knot is relatively tight such that, initially, it is immobile. Only when, driven by thermal fluctuations, sufficient contour length is introduced into the $\mathrm{KR}$, it becomes flexible enough to start its diffusion toward a chain end. We pursue a simple energetic argument to quantify the knot immobilisation. In the remainder we assume that chain relaxation is fast compared to knot immobilisation time.

After creation under high confinement the knot is not molecularly tight in the picture of de Gennes [12]. However, it is small enough such that the self-confined volume of the KR is of a diameter comparable to or smaller than $\ell_{p}$. At several contact points where the topology of the knot prevents expansion the bending stiffness causes that the respective chains lock each other, comparable to static friction effects. Fluctuations now need to simultaneously loosen a number of these locking contacts in order to allow free length to enter the KR. The energy barrier for releasing a contact is of the order of the work necessary to displace one part of the chain from the local potential minimum. To induce a displacement $\delta h$, the work $\delta E=F_{\perp} \delta h$ needs to be performed, where $F_{\perp}$ is the force acting on the contact area. To determine how $F_{\perp}$ scales with the size of the KR, $R_{\Delta}$, we note that the typical bending energy of a DNA segment of length $\ell$ and curvature radius $R_{\Delta}$ is $E_{b} \simeq \pi \kappa \ell /\left(2 R_{\Delta}^{2}\right)$, with elastic constant $\kappa=k_{B} T \ell_{p}$ [13]. As the $\mathrm{KR}$ is not molecularly tight and all segments of the KR tend to assume minimum curvature, the curvature radius is of the order of $R_{\Delta}$. A small normal in-plane displacement $\delta h$ of a stretch of DNA around the contact point changes the curvature radius approximately by $-\delta h$ (bending reduces $R_{\Delta}$ and there are no other parameters of the dimension of length in this purely geometric argument), so that $F_{\perp} \simeq d E_{b} / d(\delta h) \simeq-d E_{b} / d R_{\Delta} \sim R_{\Delta}^{-3}$. Thus, the energy necessary to unlock $n$ contacts is estimated by $\delta E_{n} \simeq n \pi(\delta h) \kappa \ell_{p} R_{\Delta}^{-3}$ if we assume $\ell$ to be of the order of the Kuhn length $\xi_{K}$. The sharp $R_{\Delta}^{-3}$-dependence of the potential barrier for loosening the KR suggests that the activation energy becomes unimportant after crossing the first few barriers, and further untightening is controlled by a different mechanism.

The fast tightening of a knot into a locked state by an external force $\zeta$ requires the crossing of practically the same potential barrier. If the associated displacement in direction of the force is $b$, the work necessary is $\delta E_{\zeta} \simeq b \zeta .\left(^{5}\right)$ The time scale for knot immobilization according to our barrier crossing argument is therefore (compare [12])

$$
\theta \simeq \tau \exp \left[\delta E_{n} / k_{B} T\right]
$$

where $\tau$ is a relaxation time, not necessarily of the entire chain. Due to the sharp $R_{\Delta}^{-3}$ dependence of the barrier $\delta E_{n}$, it rapidly decays when the size of the KR grows. Therefore the immobilization time of a knot in an initially tight configuration is large (and proportional to

$\left({ }^{4}\right) \mathrm{A}$ trefoil knot in a linear chain as well as the $\mathrm{Z}$ or $\&$ folds correspond to the bending energy of a full circle with a radius equivalent to the channel radius, approx. 50nm. Using $E \approx k_{B} T \ell_{p} \pi / R$ for a radius $R$, we find above value [13]. Higher order knots, that are likely to be created, carry higher bending energy [14].

$\left({ }^{5}\right)$ This relation is analogous to de Gennes' result for a molecularly tight knot [12]. 
the exponential of the initial tightening force $\zeta$ ), but the thermally activated untightening process starting after overcoming the initial potential barrier is extremely fast and avalanche-like. After a few thermally activated steps the knot takes its maximally released configuration, with the size restricted only by the external geometric constrains (i.e., channel or tube diameter). The next stage of the process is therefore not activation-dominated, but corresponds to a diffusion of the stored length into the KR. In a control experiment on a linear chain, it should be possible to measure $\tau$ independently. Therefore one could obtain the value of the activation energy $\delta E_{n}$ as function of the electric field smashing the knot against the channel defect, or, in the case of optical tweezers experiments, the mechanical tension $\zeta$. Note that for some of the specific knots recorded, the immobilisation period lasts for several tens of seconds. The number of contact points increases with knot complexity, while the curvature radii become somewhat smaller, such that more complex knots are expected to have a drastically enhanced immobilization time.

Confined knot. - Once unlocked by the provision of sufficient stored length, in absence of a strong line tension the knot remains in the loose mode until it escapes from the chain. By exchanging stored length between the KR and the rest of the chain, the knot performs a random walk along the polymer. As we will see, the ensuing diffusion process is biased. We distinguish two potential diffusion mechanisms, both providing the fluctuations in additional length, leading to an inchworming of the KR: (i) The first diffusive mode is characterised by a strongly disbalanced behaviour, in which length is provided to the KR by motion of the entire chain (from KR to the respective end) to either side of the knot. This 'longitudinal' mode is similar to the self-reptation picture of [9], however, our assumption takes the asymmetry into consideration, that the self-reptative diffusivity should increase when the knot gets closer to one of the chain ends. The efficiency of self-reptation should become reduced under tension (straightening of the chain). (ii) The second mode is an autonomous motion of the KR, due to the fact that it is not molecularly tight: local length fluctuations in exchange with the knot's immediate vicinity slightly change its size, allowing random directional changes. We refer to this 'transversal' mode consisting of small variations in the knot size as KR-breathing, causing a sliding motion of the KR along the chain. Once the knot arrives at one chain end, it is released, corresponding to a first exit problem with absorbing boundaries at $x_{L}=0$ and $x_{R}=0$.

Both modes of knot release imply coherent motions of considerable parts of the chain. We assume that such concerted motion of a part of the chain of length $l$ invokes the Rouse-like mobility $\mu(l) \propto l^{-1}$, so that the corresponding diffusion coefficient is $D(l)=\mu(l) k_{B} T \simeq k_{B} T / l$. Thus, if $\Delta \ll L$, KR-breathing typically involves the motion of a much shorter segment (of length $\Delta$ ) than self-reptation, unless the knot travels close to the end of the chain, when the second mode becomes comparable or even more relevant. $\left({ }^{6}\right)$ Let us now discuss the two types of motion in more detail. KR-breathing is characterised by a diffusivity

$$
D_{\mathrm{KRB}} \simeq \mu_{\mathrm{KRB}}(\mathcal{K}) k_{B} T \simeq \alpha k_{B} T / \Delta(\mathcal{K})
$$

involving the motion of a length $\approx \Delta$ of the $\mathrm{KR}$ and a small vicinal DNA. The mobility $\mu_{\mathrm{KRB}} \simeq 1 / \Delta$ will strongly depend on the knot type $\mathcal{K}$ through its size $\Delta(\mathcal{K})[9]$. In particular, $D_{\mathrm{KRB}}$ is independent of the overall chain length, $L$. In scaling terms, the diffusion time of the knot on the chain is given by $\tau_{\mathrm{KRB}} \simeq L^{2} / D \simeq \Delta L^{2}$, growing slower than the cubic scaling of the reptation time for chain dynamics in a reptation tube $[15,16]$. To obtain the dependence of the knot diffusion time on its initial location $x_{L}$ on the chain, we determine the mean first

$\left({ }^{6}\right)$ In particular, for an unconfined knot as discussed below. 
passage time $\mathcal{T}\left(x_{L}\right)$ from the diffusion equation $\partial P(x, t) / \partial t=(\partial / \partial x) D(x)(\partial / \partial x) P(x, t)$, with absorbing boundary conditions at both ends. Here, $P(x, t)$ is the probability density to find the defect at position $x$. Following Gardiner [17], $\mathcal{T}\left(x_{L}\right)$ is given by

$$
\mathcal{T}\left(x_{L}\right)=\int_{0}^{x_{L}} \frac{L_{\mathrm{red}} / 2-x^{\prime}}{D\left(x^{\prime}\right)} d x^{\prime},
$$

where $L_{\text {red }}=L-\Delta$ is the reduced length of the two linear segments of the chain. With the expression for $D_{\mathrm{KRB}}$, the diffusion time for KR-breathing yields:

$$
\mathcal{T}_{\mathrm{KRB}}\left(x_{L}\right)=\frac{\Delta L_{\mathrm{red}}^{2}}{2 \alpha k_{B} T}\left[\left(x_{L} / L_{\mathrm{red}}\right)-\left(x_{L} / L_{\mathrm{red}}\right)^{2}\right] .
$$

In the self-reptation picture, the second diffusion mode, KR motion requires rearrangement of either the left or the right chain respective to the knot location plus the KR itself. Thus,

$$
D_{\mathrm{SR}} \simeq \frac{\alpha k_{B} T}{x_{L}+\Delta}+\frac{\alpha k_{B} T}{x_{R}+\Delta}=\frac{\alpha k_{B} T(L+\Delta)}{\left(x_{L}+\Delta\right)\left(L-x_{L}\right)}
$$

that quickly increases toward either chain end. From expression (3), it follows

$$
\begin{aligned}
\mathcal{T}_{\mathrm{SR}}\left(x_{L}\right)=\frac{1}{12 \alpha k_{B} T(L+\Delta)} & {\left[x _ { L } \left\{x_{L}\left(3 L L_{\mathrm{red}}-4 L x_{L}-2 L_{\mathrm{red}} x_{L}+3 x_{L}^{3}\right)\right.\right.} \\
& \left.\left.+\Delta\left(6 L\left[L_{\mathrm{red}}-x_{L}\right]+x_{L}\left[4 x_{L}-3 L_{\mathrm{red}}\right]\right)\right\}\right]
\end{aligned}
$$

plotted in figure 2 If $\Delta \ll L$, we have $L \approx L_{\text {red }}$, and we find the simplified expression

$$
\mathcal{T}_{\mathrm{SR}}\left(x_{L}\right) \approx \frac{L^{3}}{4 \alpha k_{B} T}\left[\left(x_{L} / L\right)^{2}-2\left(x_{L} / L\right)^{3}+\left(x_{L} / L\right)^{4}\right] .
$$

Self-reptation, that is, leads to the $L^{3}$ scaling of $\mathcal{T}_{\mathrm{SR}}\left(x_{L}\right)$ in analogy to regular reptation $[15,16]$.

Combining both effects to the total diffusivity $D_{\text {diff }}\left(x_{L}\right)=D_{\mathrm{KRB}}\left(x_{L}\right)+D_{\mathrm{SR}}\left(x_{L}\right)$, we cannot obtain an analytic result for the knot diffusion time and resort to numerical evaluation. $\left({ }^{7}\right)$ Rewriting $D_{\text {diff }}=\xi_{K}^{2} / \tau_{K}$ in terms of the Kuhn length as assumed unit for stored length and therefore the fundamental random walk step size, for $D_{\text {diff }} \sim 1 \mu \mathrm{m}^{2} / \mathrm{sec}$, we find the associated step time $\tau_{K} \sim 10^{-2}$ sec. From above relations we obtain the graphs in figure 2 For initial positions of the KR close to a chain end, the mean first passage times for both diffusion mechanisms becomes identical, as it should. For a chain of the size of the $\lambda$ DNA ( $L=165 \xi_{K}$ ), the individual modes are both relevant. The scaling of $\mathcal{T}_{\text {diff }}$ with the chain length for the symmetric initial condition $x_{L}=L_{\text {red }} / 2$ is shown in figure 2 demonstrating that for longer chain lengths, KR-breathing dominates. The scaling dependence of the overall knot diffusion time provides a direct way to test our model experimentally. It will be interesting to monitor the time series of knot defects in a large ensemble of realizations to obtain statistically relevant results for the two contributions $\theta$ and $\mathcal{T}_{\text {diff }}$, i.e., immobilisation and diffusion times.

Flexible chain. - For flexible chains with persistence lengths much smaller than the channel diameter, the $\mathrm{KR}$ is allowed to expand. The size of (at least, simpler) knots in absence of tension, or under moderate tension scales like a power-law of the chain length, $\ell_{k} \simeq a^{1-c} L^{c}$, with $c \approx 0.7[18,19]$. The modus operandi of knot escape from the chain can now follow two different tracks: (i) First, the knot can diffuse out while being localized at all

$\left({ }^{7}\right)$ Noting that in reference [9], for a slightly pulled chain the diffusivity $D_{\text {diff }} \approx 1.2 \mu \mathrm{m}^{2} / \mathrm{sec}$ was measured. 

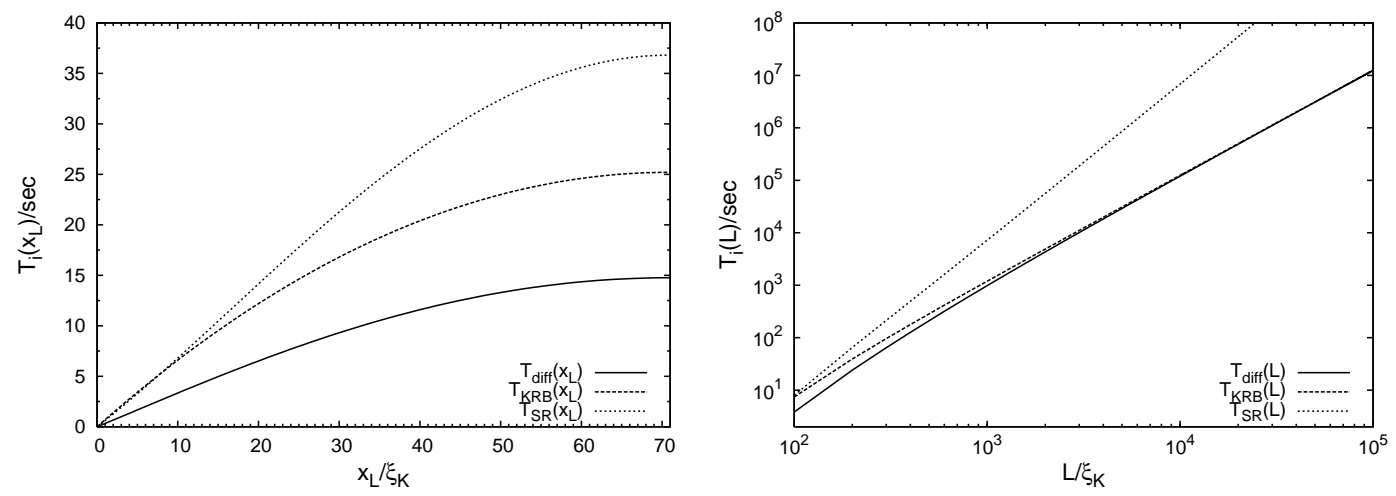

Fig. 2 - Mean first passage time $\mathcal{T}\left(x_{L}\right)$ versus initial position $x_{L}$ of the knot for fixed chain length $L=165 \xi_{K}$ (left), and versus chain length $L$ for symmetric initial condition $x_{L}=L_{\mathrm{red}} / 2=71 \xi_{K}$ (right). With $\Delta=23 \xi_{K}$ and $\tau_{K}=10^{-2}$ sec.

times, corresponding to the scenario developed above. As $\xi_{K}$ is much smaller and therefore $L$ much larger in units of $\xi_{K}$, KR-breathing dominates, and the first passage time scales like $\mathcal{T}_{\text {diff }} \simeq L^{2} / D_{\text {KRB }}$. Without effective confinement by the channel walls, the KR grows with $L$, entering the diffusivity $D_{\mathrm{KBR}}=D_{\mathrm{KBR}}(L)$, i.e., the diffusive component with fractal scaling $\mathcal{T}_{\mathrm{KRB}} \simeq L^{2+\alpha}$ yields. For knots dominated by finite size effects (or complex knots that might tend to delocalisation) [20], or under dense or $\theta$ conditions [21], the KR scales like the overall chain length, and we would obtain the reptation scaling $\mathcal{T}_{\mathrm{KRB}} \sim \mathcal{T}_{\text {diff }} \simeq L^{3}$. Conversely, when confined to two dimensions by adhesion to a surface or by sandwiching between two parallel walls, the knot localises tightly and $\alpha=0$ [10]. (ii) Second, in absence of external tension the knot can be undone by swelling of the KR until it is of the size $L$ of the chain, and the chain ends are inside the KR. This mode is expected to scale like the all-chain Rouse time $\simeq L^{3}$.

Discussion. - The possibility to devise nanochannels of a width comparable to the persistence length of polymers, and to monitor the end-to-end distance of a single polymer is a fine example how new experimental techniques can probe mechanically domains of fundamental behaviour of single polymers. We presented a simple scaling model of both the immobilisation and the diffusion of knots in DNA confined in nanochannels, identifying two different reptation modes. While self-reptation has been considered earlier, we propose a modified self-reptation picture that is, in fact, asymmetric. We also suggest a new mode, namely, KR-breathing, that is expected to dominate the knot diffusion in longer chains. By varying chain length $L$ and the channel diameter-to-persistence length ratio, we expect future experiments to provide new insight in the reptation of single chains that self-confine themselves.

It appears obvious that when the knot gets close to one of the chain ends, the close end can easily reptate into the KR and subsequently release the knot, without the far end being involved. In this picture, it is not necessary to have full chain equilibration on time scales slower than the diffusion time. The connected asymmetry of the diffusion coefficient with respect to the knot position along the chain is indeed corroborated by recent simulations results of a trefoil knot diffusing on a linear chain under moderate tension [22]. The existence of two diffusion modes makes sure that the knot mobility is finite even for very long chains, $L \rightarrow \infty$. In other pictures assuming relaxation of the whole chain as relevant diffusion time scale, in a very long polymer the knot would become effectively immobile.

We note that small external stretching forces should not affect the knot diffusion modes. 
Moderate forces are expected to reduce self-reptation; as long as the blob size remains larger than the KR, KR-breathing should not be disturbed. For high forces, eventually the system will be immobilised according to the above picture. We also note that we assumed that the diffusion of the knot modes is Brownian. While this is always true for the KR-breathing mode, for long chains self-reptation should become subdiffusive according to arguments brought forth in the discussion of nanopore translocation [23,24]. In that case, KR-breathing becomes even more dominant. It would be very interesting to directly observe subdiffuion in experiments.

Lastly, from the experimental side, it appears that the de Gennes hypothesis regarding knot immobilization time (Eq. 11) could be tested by varying the field strength used to compress the DNA, as the tightening force should be related to the compression field. The de Gennes hypothesis would suggest that the knot immobilization time is a strongly increasing function of the compression field. Also, it would be interesting to study how the generated knot spectrum depends on compression field strength and duration. This could easily be accomplished by compressing DNA at varying field strengths.

RM acknowledges the Natural Sciences and Engineering Research Council (NSERC) of Canada and the Canada Research Chair program for support. For the experimental work, which was carried out at Prof. Austin's group at Princeton, we would like to acknowledge support from NIH (HG01506) and NSF Nanobiology Technology Center (BSCECS9876771).

\section{REFERENCES}

[1] OdiJk, T., Macromol., 16 (1983) 1340.

[2] Tegenfeldt, J. O., Proc. Natl. Acad. Sci. USA, 101 (2004) 10979.

[3] Reisner, W. et al., Phys. Rev. Lett., 94 (2005) 196101.

[4] Austin, R., Nat. Mat., 2 (2003) 611.

[5] Reisner, W. et al., Unpublished.

[6] Deibler, R. W., Rahmati, S., and Zechiedrich, E. L., Genes \& Developm., 15 (2001) 748.

[7] Stazcek, P., and Higgins, N. P., Mol. Microbiol., 29 (1998) 1435.

[8] Arsuaga, J. et al., Proc. Natl. Acad. Sci. USA, 99 (2002) 5373.

[9] Bao, X. R., Lee, H. J., and Quake, S. R., Phys. Rev. Lett., 91 (2003) 265506.

[10] Metzler, R. et al., Phys. Rev. Lett., 88 (2002) 188101.

[11] Vologodskit, A., Biophys. J., 90 (2006) 1594.

[12] De Gennes, P.-G., Macromol., 17 (1984) 703.

[13] Marko, J. F., and Siggia, E. D., Phys. Rev. E, 52 (1995) 2912.

[14] Stasiak, A., Katritch, V., and Kauffman, L. H. (Editors), Ideal Knots (World Scientific, Singapore) 1998.

[15] De Gennes, P.-G., J. Chem. Phys., 55 (1971) 572.

[16] Doi, M., and Edwards, S. F., The Theory of Polymer Dynamics (Clarendon Press, Oxford, UK) 1986.

[17] Gardiner, C. W., Handbook of Stochastic Methods for Physics, Chemistry and the Natural Sciences (Springer, Berlin) 1996.

[18] Virnau, P., Kantor, Y., and Kardar, M., J. Am. Chem. Soc., 127 (2005) 15102.

[19] Marcone, B. et al., J. Phys. A, 38 (2005) L15.

[20] Grosberg, A. Yu., Feigel, A., and Rabin, I., Phys. Rev. E, 54 (1996) 6618.

[21] Hanke, A. et al., Euro. Phys. J., 12 (2003) 347.

[22] Arend, A., Rzehak, R., and Zimmermann, W., Preprint.

[23] Kantor, Y., and Kardar, M., Phys. Rev. E, 69 (2004) 021806.

[24] Metzler, R., and Klafter, J., Biophys. J., 85 (2003) 2776. 